\title{
A New Optical TDM Ring Architecture
}

\author{
An Chen, Chin-Tau Lea, and Albert Kai-Sun Wong
}

\begin{abstract}
In this paper, we explore new optical time-division multiplexing (OTDM) ring architectures. The objective of our study is to achieve high space-reuse efficiency of each time slot without using time-slot interchanges, which are expensive and difficult to implement in the optical domain. Although a TDM slot is often compared to a wavelength channel in a wavelength-division multiplexing (WDM) network, we show that the intrinsic propagation delay in an OTDM network will lead to a phenomenon, called a slot cycle in the paper, that has no counterpart in WDM networks. By exploring the concept and the algebraic relationship between the frame size and the total propagation delay of the ring, we discover a novel OTDM ring architecture that achieves a high space-reuse efficiency without using time-slot interchanges. The equivalent of this architecture in the WDM domain can only be achieved with the use of many wavelength converters. We also study the efficiency of the architecture based on different types of traffic demand models in the network.
\end{abstract}

Index Terms-Optical ring architecture, optical time-division multiplexing (TDM).

\section{INTRODUCTION}

W AVELENGTH-DIVISION multiplexing (WDM) systems have already been widely used in the field. However the offered bandwidth granularity is higher than many applications. An optical time-division multiplexing (OTDM) system [1]-[4] divides each wavelength into multiple time slots, and each time slot offers a finer bandwidth granularity. OTDM requires minimum header processing and is considered a more practical form of time-domain optical switching in the near future than packet switching [5]-[7], which is hindered by the lack of adequate optical processing devices.

Ring is the most commonly used topology for TDM systems, i.e., SONET rings, and it is our focus in the paper. Data are organized into repetitive frames and a data channel is identified by the allocated time slot in the frame. Each node can perform add/drop operations by inserting and removing data from a slot of the ring. Sometimes a node needs to move the data from an incoming slot to any outgoing slot. This switching function is performed by a time-slot interchange (TSI). An electronic TSI is usually implemented with random access memory. But an optical TSI is much harder to implement. One example is given in Fig. 1 where the optical TSI consists of an optical switch and

Paper approved by A. Pattavina, the Editor for Switching Architecture Performance of the IEEE Communications Society. Manuscript received December 14, 2005; revised May 15, 2006 and December 20, 2006. This work was supported in part by the HKRGC under Grant HKUST 6201/03E and in part by the ITF under Grant GHS-025-04. This paper was presented in part at the IEEE International Conference on Communications (ICC'06), Istanbul, Turkey, June 2006.

C.-T. Lea and A. K.-S. Wong are with the Department of Electronic and Computer Engineering, Hong Kong University of Science and Technology, Kowloon, Hong Kong (e-mail: eelea@ece.ust.hk; eealbert@ece.ust.hk).

A. Chen is with Giant Innovation, Hong Kong (e-mail: anchen@ece.ust.hk).

Digital Object Identifier 10.1109/TCOMM.2007.908521

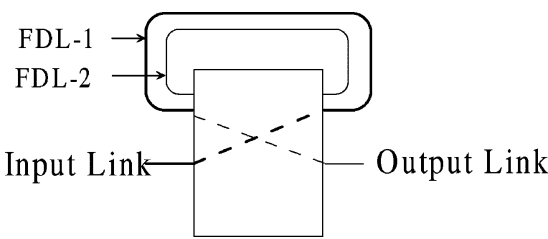

Fig. 1. Optical TSI example which consists of an optical space switch and FDLs.

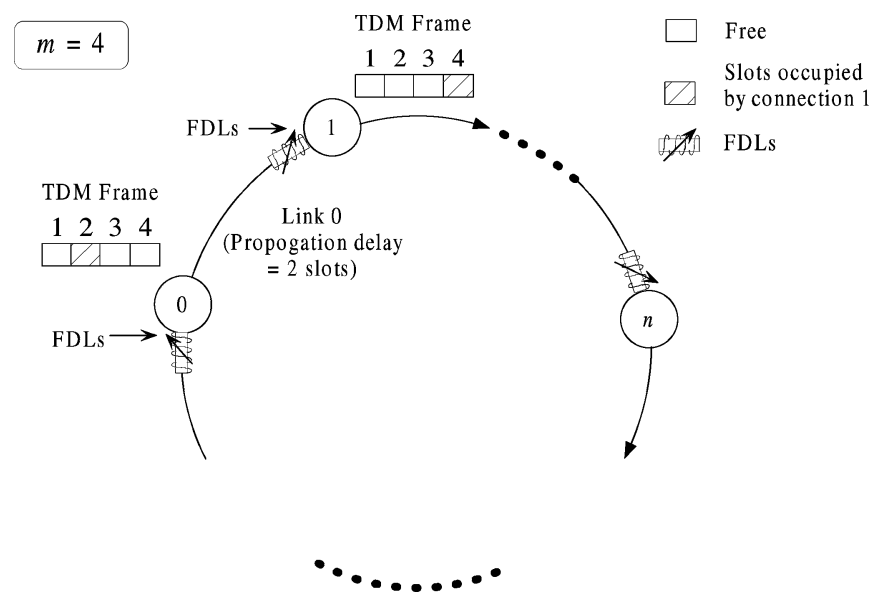

Fig. 2. Ring without TSI needs only simple fiber delay lines in each node to synchronize the incoming and outgoing slots. It is much easier and cheaper to build than a ring with TSI.

fiber delay lines. A data block will pass through the switch a variable number of times, depending on how long it will stay in the buffer, and the signal attenuation will not be a constant. It is particularly severe if the TDM frame size is large and a data block needs to stay in the buffer for many time slots. Also, the size of the buffer grows with the OTDM frame size, which is usually large. The complexity and the cost of the TSI will make an OTDM ring impractical to build for the current time being.

If we remove TSIs, an OTDM ring only needs delay lines in each node to synchronize the incoming and outgoing slots (Fig. 2). This can reduce the complexity and cost significantly. Given today's optical buffer technology [8], [9], the architecture in Fig. 2 is perhaps the only practical OTDM ring architecture for the time being. The problem, however, is that the space-reuse efficiency of the ring is poor. Consider the example in Fig. 3(a). To simplify the discussion, we assume the propagation delay between two nodes to be zero (or a multiple of the frame size) such that slot 0 sent out by node $i$ will be sent out again as slot 0 by node $(i+1)$. Suppose slot 0 from node 1 to node 7 has already been occupied by an existing lightpath. Now, a new lightpath from node 7 to node 2 needs to be set up. As the two 


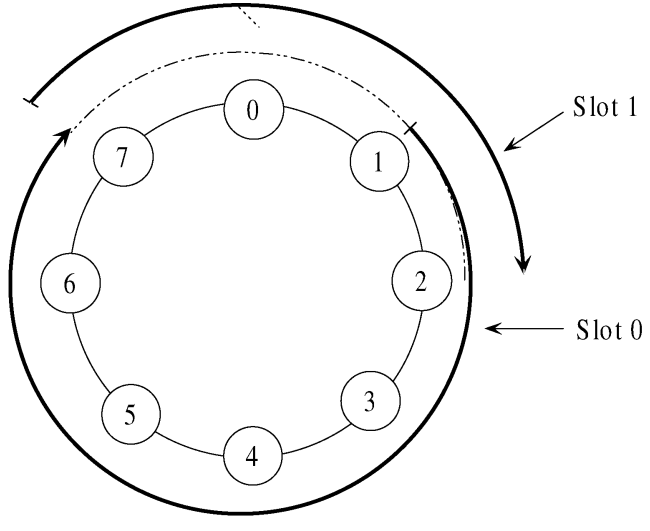

(a)

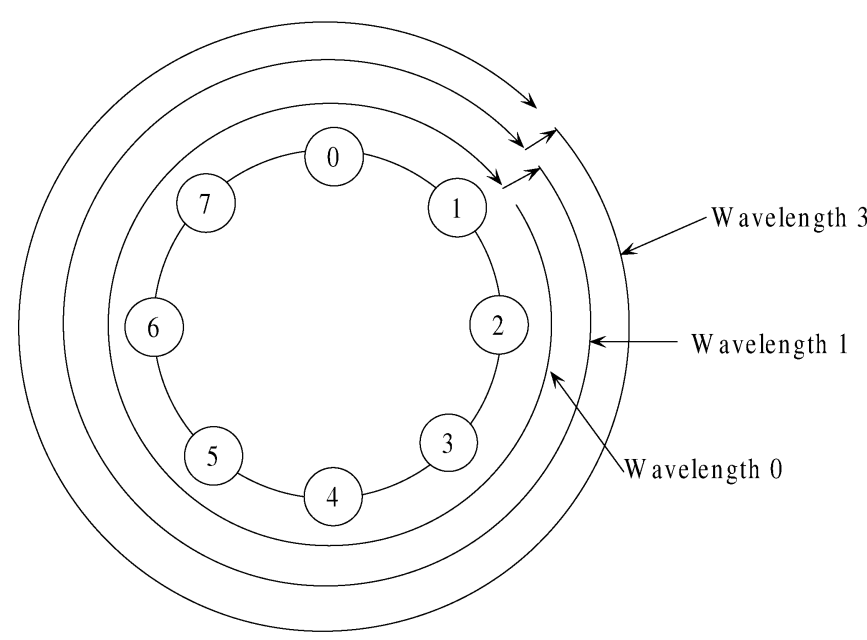

(b)

Fig. 3. (a) One lightpath from node 1 to node 7 has been set up. If we intend to set up another lightpath from node 7 to node 2, we have to use a different slot because the two paths overlap in the segment between nodes 1 and 2. (b) The same space-reuse efficiency problem also occurs in a WDM ring. One way to improve the space-reuse efficiency is to place each wavelength converter at only one node (node 1 in this figure) to connect all wavelengths into a large cycle. The signal of wavelength 1 will be converted to that of wavelength 2 when it cycles back to node 1 , and wavelength 2 will be converted to wavelength 3 , etc. The result is a larger wavelength cycle and a better space-reuse efficiency

lightpaths overlap in the segment between node 1 to node 2 , we must use a different slot for the new connection. In other words, the remaining segment of slot 0 between node 7 and node 1 cannot be used for the second lightpath even if it is available.

A similar space-reuse efficiency problem also occurs in a WDM ring where each wavelength corresponds to one slotslot 0 is replaced by wavelength 0 and slot 1 is replaced by wavelength 1 , etc. If we need to convert signals of any wavelength to signals of any other wavelength, we need to put $m$ wavelength converters in each node, where $m$ is the number of wavelengths (or the frame size in an OTDM network). If there are $N$ nodes in the ring, we need $N m$ converters. This is obviously too expensive to do. Instead, we can equip only one node with wavelength converters and make a larger wavelength cycle. For example, if we have only one wavelength converter, we can connect the wavelengths 0 and 1 into a longer cycle and achieve a better space-reuse efficiency [Fig. 3(b)]. If we use $m$ wavelength converters (at one node only), we can connect all wavelengths into one large cycle and improve the space-reuse efficiency even further [10], [11].

If we put a TSI in one node of an OTDM network, we can achieve a similar result as in Fig. 3(b) due to the slot/wavelength equivalence. But this is not needed. We discover that in spite of the slot/wavelength equivalence, there is a fundamental difference-from system point of view-between the two types of networks (see Section II). By exploring this difference and the algebraic relationship between the TDM frame size and the total propagation delay of the ring, we can build an OTDM ring with the same capability as described in Fig. 3(b) without using any optical TSIs. This will greatly reduce the cost and simplify the design of an OTDM ring.

We will evaluate the space-reuse efficiency of the architecture through some routing and time-slot assignment (RTA) algorithms to be discussed later in the paper. The problem bears some similarities to the routing and wavelength assignment (RWA) problem [10]-[12] in the WDM rings. In [12], the authors considered the RWA problem in a unidirectional ring under a traffic matrix of which the total number of lightpaths passing through a link is bounded. The goal is to minimize the number of wavelengths required for a given load bound. The RWA problem for a bidirectional ring under a different traffic model was also considered in [10] and [11]. The authors determined the minimum wavelength requirements for lightpath requests in $N$ node $P$ transceivers networks (all $P N$ requests are received simultaneously). The techniques used in [10]-[12] provide a useful reference for our study. But the fundamental difference introduced by the propagation delay, as mentioned previously, will make our approach different from that used for WDM rings.

The rest of the paper is organized as follows. In Section II, we discuss the fundamental properties of a TSI-free OTDM ring and propose a new OTDM ring architecture. The major innovation of the architecture is that it can achieve great space-reuse efficiency without using optical TSIs. This significantly simplifies the design. In Sections III and IV, we study the space-reuse efficiency of the TSI-free OTDM rings presented in the paper under two different traffic models. We conclude the discussion in Section V.

\section{NEw OpticAl TDM Ring ARchitecture}

We present a novel OTDM ring architecture without using TSIs. The properties presented in this section are general and apply to all TSI-free OTDM rings. We first consider a unidirectional TDM ring and bidirectional rings are considered in Section III. There are $N$ nodes in the ring, and we number the nodes in the increasing order from 0 to $N-1$ in the clockwise direction. Each node in the ring can support multiple wavelengths and data of each wavelength are organized into TDM frames. Our discussion focuses on the TDM properties of just one wavelength. A lightpath will occupy a slot on different links of the ring. Once the lightpath is set up, subsequent data packets will continue to use the same time slots reserved on each link. 


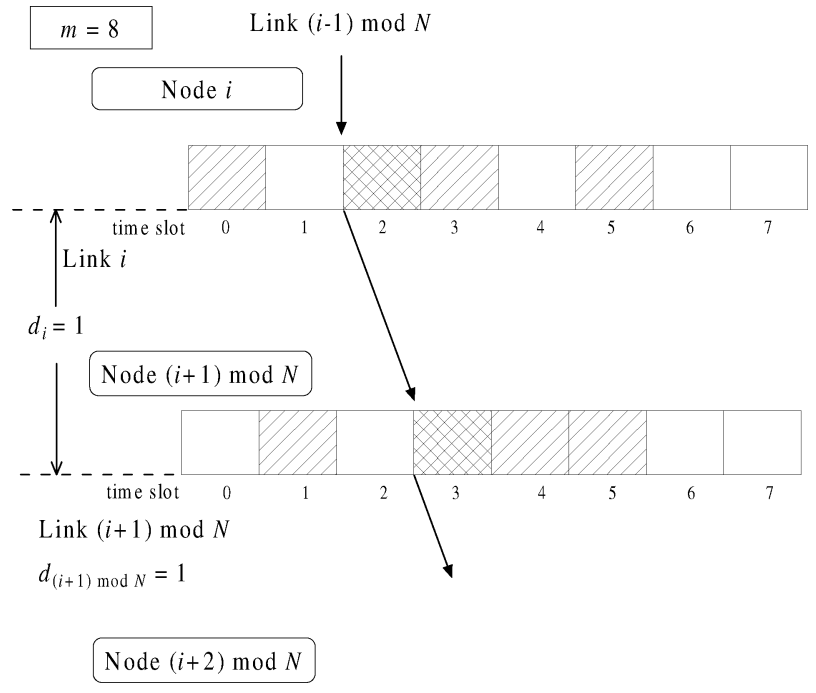

Fig. 4. Propagation delays encountered by a photonic circuit.

In the paper, we assume that the slot size is 1 and the frame size is $m$. The link between node $i$ and its next node neighbor $[(i+1) \bmod N]$ is denoted by $i$ (Fig. 2) and the propagation delay on link $i$ is denoted by $d_{i}$. Let $D$ be the total propagation delay of the ring and $D=d_{0}+d_{1}+\cdots+d_{N-1}$. We assume each node is equipped with a simple fiber delay line to make $d_{i}$ an integer (i.e., a multiple of time slots). This is to synchronize the transmission time of the outgoing slots with the arrival time of the incoming slots. Without loss of generality, we can assume that all nodes begin their frames at the same time, and slot 0 is transmitted from all nodes simultaneously. In a TSI-free OTDM ring, a slot number in an incoming frame will change, due to the propagation delay, to a different slot number in the outgoing frame. For example in Fig. 4, slot 2 in the incoming frame at node $i$ is changed to slot number 3 in the incoming frame at node $[(i+1) \bmod N]$ due to the one-unit propagation delay (the shaded time slots on the links are time slots that are already in use). Slot-number shift caused by the nonzero propagation delay is a major characteristic of an OTDM ring that has no counterpart in a WDM ring without wavelength converters-a data block will always be carried by the same wavelength.

We can use $\left(n_{i}, s_{i}\right)$ to identify a slot on the ring where $s_{i}$ is the slot number inside the frame that is located on the egress link of node $n_{i}$. As the ring does not use TSIs, we have the following relationship.

$$
\left(n_{i}, s_{i}\right)=\left(\left(n_{i-1}+1\right) \bmod N,\left(s_{i-1}+d_{n_{i-1}}\right) \bmod m\right) .
$$

We can trace a slot as it changes its slot number along the ring and obtain a sequence of slot numbers. This sequence is called a track in the paper. A track $k$, denoted by $T^{k}$, can be written as

$$
\begin{aligned}
T^{k} & =\left\{\left(n_{0}^{k}, s_{0}^{k}\right),\left(n_{1}^{k}, s_{1}^{k}\right), \ldots,\left(n_{L}^{k}, s_{L}^{k}\right) \mid n_{i}^{k}\right. \\
& =\left(n_{i-1}^{k}+1\right) \bmod N \text { and } s_{i}^{k}=\left(s_{i-1}^{k}+d_{n_{i-1}^{k}}\right) \bmod m, \\
& 0<i \leq L\}
\end{aligned}
$$

where the slots $\left(n_{i-1}^{k}, s_{i-1}^{k}\right)$ and $\left(n_{i}^{k}, s_{i}^{k}\right)$ satisfy (1). The length of track $T^{k}$, denoted by $\left|T^{k}\right|$, is the number of hops the track spans. For example, the length of $T^{k}$ in (2) is $(L+1)$. The following property states that if two tracks pass a link in different time slots, then they will not occupy the same slot at the next $(N-1)$ hops.

Property 1: $T^{1}$ and $T^{2}$ are two tracks. Assume slot $\left(n_{i}^{1}, s_{i}^{1}\right) \in$ $T^{1}$ and slot $\left(n_{j}^{2}, s_{j}^{2}\right) \in T^{2}$. If $n_{i}^{1}=n_{j}^{2}$ and $s_{i}^{1} \neq s_{j}^{2}$, then the following must hold

$$
n_{i+l}^{1}=n_{j+l}^{2} \text { and } s_{i+l}^{1} \neq s_{j+l}^{2}, \quad \text { for } 0<l<N .
$$

Proof: This obviously will hold because of (1).

The following property shows that if a track continues, it will eventually form a cycle.

Property 2: Suppose that track $k$ starts from $\left(n_{0}^{k}, s_{0}^{k}\right)$ and continues along the ring until it first reaches a slot, say $\left(n_{j}^{k}, s_{j}^{k}\right)$, which already belongs to the track. Then, $n_{j}^{k}=n_{0}^{k}$ and $s_{j}^{k}=s_{0}^{k}$.

Proof: We prove it by way of contradiction. Assume $n_{j}^{k}=n_{i}^{k}$ and $i>0$. By Property $1,\left(n_{j-1}^{k}, s_{j-1}^{k}\right)=\left(n_{i-1}^{k}, s_{i-1}^{k}\right)$ must be true. Thus, $\left(n_{j}^{k}, s_{j}^{k}\right)$ cannot be the first slot that is repeated in the track. This is a contradiction. Therefore, $n_{j}^{k}=n_{0}^{k}$ and $s_{j}^{k}=s_{0}^{k}$ must hold.

The cycle formed by tracing a slot is called a slot cycle. Two slot cycles $S^{1}$ and $S^{2}$ are said to be equal, denoted by $S^{1} \equiv S^{2}$, if slot $\left(n_{i}, s_{i}\right) \in S^{1}$, then $\left(n_{i}, s_{i}\right) \in S^{2}$ and vice versa. From the definition, the starting slot numbers of two equal slot cycles need not be the same. Two slot cycles $S^{1}$ and $S^{2}$ are said to be disjoint if $S^{1}$ and $S^{2}$ do not share any common slot. Define the universal set $U$ as the set of all slots on the ring; that is

$$
U=\{(n, s) \mid 0 \leq n<N \text { and } 0 \leq s<m\} .
$$

The following property says that all different slot cycles must be disjoint.

Property 3: All slots in a TDM ring network can be divided into disjoint slot cycles; that is

$$
\bigcap_{k=1}^{K} S^{k} \text { and } S^{i} \cap S^{j}=\phi, \quad \text { for all } 0<i, j \leq K \text { and } i \neq j .
$$

Proof: We start from any slot and trace the associated slot numbers along each link until a slot cycle is formed. If there are any slots left, we repeat the same process until we exhaust all the slots. From Property 1, all the slot cycles must be disjoint.

The main results of the paper are Properties 4 and 5 given later. They show that by exploiting the algebraic relationship between the frame size $m$ and the total delay $D$, we can design a TSI-free OTDM ring with any slot-cycle length.

Property 4: All slot cycles in a TDM ring network have the same length $L=x N$, where $x$ is the smallest integer that satisfies $(x D \bmod m=0)$, where $m$ is the frame size and $D$ is the total delay of the ring.

Proof: Suppose track $k$ starts from $\left(n_{0}^{k}, s_{0}^{k}\right)$ and continues along the ring until it first reaches a slot, say $\left(n_{i}^{k}, s_{i}^{k}\right)$, which equals $\left(n_{0}^{k}, s_{0}^{k}\right)$ (see Property 2). Since $n_{i}^{k}=n_{0}^{k}$, we have $\left(n_{0}^{k}+i\right) \bmod N=n_{i}^{k}$. Therefore, $i=x N$, where $x$ is an integer and represents the number of times the cycle passes through 


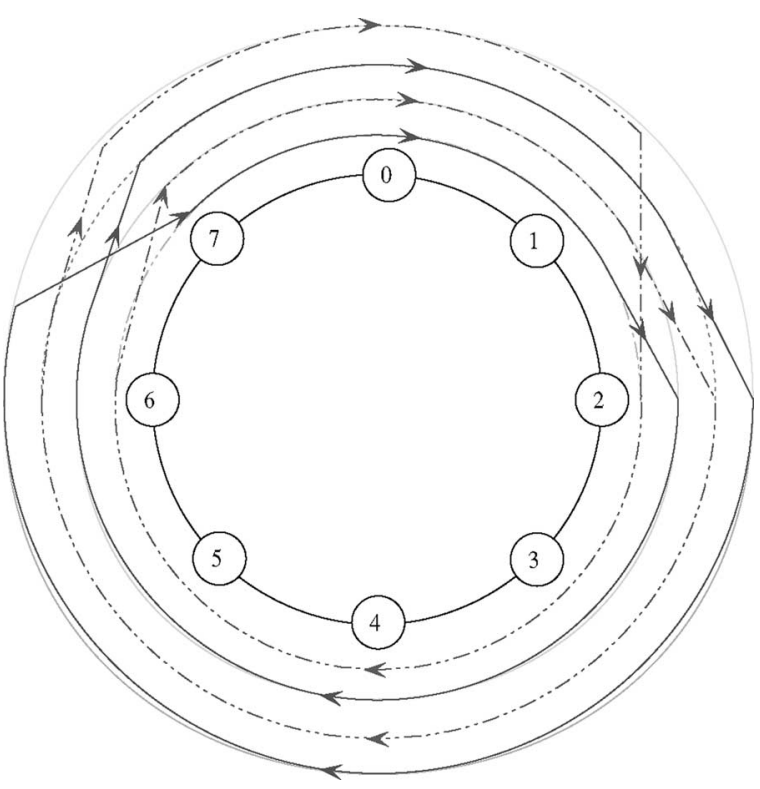

Fig. 5. There are 2 slot cycles assuming the propagation delays are 4 slots except link 1 and 6 (both have delay of 1 slot).

node $n_{0}^{k}$. Since

$$
\begin{aligned}
s_{i}^{k} & =\left(s_{0}^{k}+\sum_{j=0}^{i-1} d_{n_{j}^{k}}\right) \bmod m \\
& =\left(s_{0}^{k}+x \sum_{j=0}^{N-1} d_{j}\right) \bmod m \\
& =\left(s_{0}^{k}+x D\right) \bmod m
\end{aligned}
$$

we have

$$
x D \bmod m=0 .
$$

For the first repeat of $\left(n_{0}^{k}, s_{0}^{k}\right), x$ must be the smallest integer satisfying (3).

One example is shown in Fig. 5 where $m=4, N=8$, and the propagation delay $=4$ slots on links between any two nodes except the link between nodes 1 and 2 , and the link between nodes 6 and 7 (both have a propagation delay of 1 slot). There are only two slot cycles in the ring; one is shown in a solid line and the other in a dashed line. Both cycles have the same length of 16 hops.

Property 5: If $m$ (the frame size) and $D$ (total delay) are relatively prime, the TDM ring has only one slot cycle with length $m N$.

Proof: From Property 4, the length of every slot cycle is $x N$, where $x$ is the smallest integer that satisfies $(x D \bmod m=0)$. If $D$ and $m$ are relatively prime, then we have $x=m$ from (3). Thus, the cycle length is $m N$. This is possible only if all slots belong to this cycle.

We will study the impact of cycle lengths on the performance of the proposed TSI-free OTDM rings in Sections III and IV using two different traffic models. Under most circumstances, the longer the cycle length, the higher the space-reuse efficiency.
Properties 4 and 5 indicate that we can exploit the algebraic relationship between $m$ and $D$ to design a TSI-free OTDM ring with any slot-cycle length. This applies to a bidirectional ring (one clockwise and one counterclockwise) as well. Suppose the bidirectional ring is a single slot-cycle ring and we like to keep the same property in case of failures. This can be easily achieved if we choose a prime $m$. For example, suppose we have $N=6$, the delay between any two nodes $=2$, and $m=13$. When one link fails and the two rings are joined, the equivalent topology for the healed ring has $N=10, D=20$, and $m=13$. According to Property 5, the healed ring still contains only one cycle.

\section{Space-Reuse EfFiciency: Traffic Model I}

We compare space-reuse efficiencies of TSI-free OTDM rings with different slot-cycle lengths in this and the next section. Let $T=\left[t_{i, j}\right]$ be the traffic demand matrix, where $t_{i, j}$ is the number of requested lightpaths from node $i$ to node $j$. Each lightpath is realized in the time domain by finding a free track between the source and destination nodes. Note that different traffic matrices can lead to different results. We will select two types of traffic matrices that have been widely used in the literature.

We first consider a single-port pattern [10], [11] in this section where each node can only originate and terminate at most one lightpath, and each lightpath has the capacity of one time slot (i.e., $t_{i, j} \leq 1$ ). Lightpaths to be established will form a logical topology on top of the physical OTDM ring. Every singleport traffic pattern can be specified by a permutation. Since a permutation consists of one or multiple permutation cycles, the logical topology formed by single-port traffic patterns will be a loop or multiple loops (Each permutation cycle represents a logical loop, and we do not use the word "ring" to prevent confusions with the physical OTDM ring). For example, suppose $N=8$. Then, the connection pattern

$\begin{array}{lllllllll}\text { From } & 0 & 1 & 2 & 3 & 4 & 5 & 6 & 7 \\ \text { To } & 7 & 0 & 5 & 2 & 3 & 1 & 4 & 6\end{array}$

has one permutation cycle and the logical topology is a single loop: node $0 \rightarrow$ node $7 \rightarrow$ node $6 \rightarrow$ node $4 \rightarrow$ node $3 \rightarrow$ node $2 \rightarrow$ node $5 \rightarrow$ node $1 \rightarrow$ node 0 .

The following connection pattern

$$
\begin{array}{lllllllll}
\text { From } & 0 & 1 & 2 & 3 & 4 & 5 & 6 & 7 \\
\text { To } & 7 & 0 & 5 & 4 & 2 & 3 & 1 & 6
\end{array}
$$

has two permutation cycles and the corresponding logical topology is two loops:

node $0 \rightarrow$ node $7 \rightarrow$ node $6 \rightarrow$ node $1 \rightarrow$ node 0 (1st loop)

node $2 \rightarrow$ node $5 \rightarrow$ node $3 \rightarrow$ node $4 \rightarrow$ node 2 (2nd loop).

In the following, we compare space-reuse efficiencies based on single-port traffic patterns that have only one loop [10]. With this assumption, we can derive close-form results for the problem under study. We consider bidirectional rings-one clockwise and one counterclockwise. We also assume the cycle lengths of the two rings to be the same. We call the two lightpaths $n_{k} \rightarrow n_{j}$ and $n_{j} \rightarrow n_{h}$ adjacent. In other words, two lightpaths are adjacent if there is no gap between the destination 


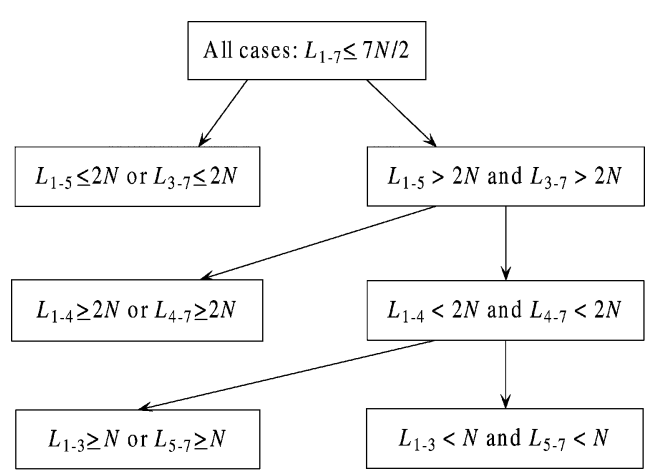

(a)

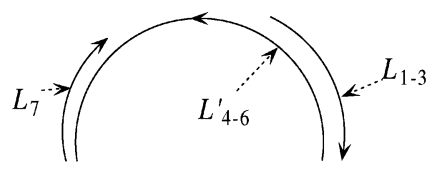

(b)

Fig. 6. (a) All the cases considered in the proof of Property 9. (b) The case of $L_{1-3}<N$ and $L_{5-7}<N$ in (a).

node of the first lightpath and the source node of the second lightpath. Similarly, we call the following $(k-1)$ connections adjacent: $n_{1} \rightarrow n_{2}, n_{2} \rightarrow n_{3}, \ldots, n_{k-2} \rightarrow n_{k-1}, n_{k-1} \rightarrow n_{k}$.

Property 6: Assume the length of all slot cycles is $N$ in a bidirectional ring. A single-loop logical topology (consisting of all nodes) can be constructed if $m \geq\lceil N / 3\rceil$, where $m$ is the frame size of the bidirectional OTDM ring.

Proof: Under this condition (cycle length $=N$ ), the time slot assignment and the routing (clockwise or counterclockwise) will be equivalent to that in a WDM ring as given in [10]. The proof is omitted here.

Property 7: Given a set of $K$ adjacent connections, if we use $q$ hops to set them up in the clockwise ring, then we can use $(K N-q)$ hops to set them up in the counterclockwise ring.

Proof: Consider one lightpath only. If it can be set up with $r$ hops in one direction, then it can be established with $(N-r)$ hops in the reverse direction. Thus we have the property for $K$ adjacent connections.

Property 8: A set of $K$ adjacent connections can be fit into a slot-cycle of length $\lfloor K N / 2\rfloor$ of a unidirectional ring.

Proof: In Property 7, either $q$ or $(K N-q)$ is $\leq\lfloor K N / 2\rfloor$. We select the smaller one of the two to set up the $K$ adjacent lightpaths.

Property 9: A set of 7 adjacent lightpaths can be fit into a slot-cycle of length $2 N$ of a bidirectional ring (both directions are used).

Proof: Denote these seven adjacent connections by 1 to 7 . By Property 8, we can set up the 7 connections along one direction with the total hop-length $\leq 7 N / 2$, and in the other direction with the total hop length $\geq 7 N / 2$. Without loss of generality, we assume that the hop length of the clockwise direction is $\leq 7 N / 2$. We can further divide the cases to be considered as shown in Fig. 6(a). In each case, we show that all seven adjacent connections can be fit into one slot cycle of length $2 N$ in a bidirectional ring (both directions are used).
The proof starts with the consideration of the first and last 5 adjacent lightpaths.

I) $L_{1-5} \leq 2 N$ or $L_{3-7} \leq 2 N$ : Route the first five (or last five) lightpaths into the slot-cycle in the clockwise direction. We then place the remaining two lightpaths in the opposite direction and this is always possible because each lightpath requires a hop length $<N$.

II) $L_{1-5}>2 N$ and $L_{3-7}>2 N$ : we divide them into two sub-cases:

1) $L_{1-4} \geq 2 N$ or $L_{4-7} \geq 2 N$ : When $L_{1-4} \geq 2 N$, it is obvious that $L_{5-7} \leq 3 N / 2$ (since $L_{1-7} \leq 7 N / 2$ ) and $L_{1-4}^{\prime} \leq 2 N$ (since $\left.L_{1-4}+L_{1-4}^{\prime}=4 N\right)$. We can route the last 3 lightpaths in the clockwise direction and place the remaining lightpaths in the opposite direction. A similar discussion applies to the case $L_{4-7} \geq 2 N$.

2) $L_{1-4}<2 N$ and $L_{4-7}<2 N$ : We again divide this case into two sub-cases based on first and last 3 adjacent lightpaths:

2.1) $L_{5-7} \geq N$ or $L_{1-3} \geq N$ : When $L_{5-7} \geq N$, clearly $L_{5-7}^{\prime} \leq 2 N$. Therefore, we can route the first 4 lightpaths in the clockwise direction and place the remaining lightpaths in the opposite direction. A similar construction can be said for the case $L_{1-3} \geq N$.

2.2) $L_{1-3}<N$ and $L_{5-7}<N$ : We firstly route lightpaths 1-3 in the clockwise direction. Since $L_{4-7}<2 N$, we get $L_{4-7}^{\prime}>2 N$. The remaining lightpaths cannot fit in the slot-cycle in the counter-clockwise direction. However since $L_{1-5}>2 N$ and $L_{1-3}<N$, we get $L_{4-5}>N$ and $L_{4-5}^{\prime}<N$. It is obvious that $L_{4-5}^{\prime}+L_{6}^{\prime}<$ $N+N=2 N$. We route 4-6 in the counterclockwise direction. Then we have only one choice: route lightpath 7 in the clockwise direction. This is possible only if the following condition holds: $L_{1-3}+L_{7}<L_{4-6}^{\prime}$ [see Fig. 6(b)]. We show below this is indeed the case.

Since $L_{4-7}^{\prime}>2 N, L_{7}^{\prime}<N$, we get $L_{4-5}^{\prime}+L_{6}^{\prime}>N$. Let $L_{1-3}=N-\theta$ and $L_{4-6}^{\prime}=N+\beta$, where $0<\theta, \beta<N$. Since $L_{4-7}^{\prime}>2 N$ and $L_{4-6}^{\prime}=N+\beta$, we have $L_{7}^{\prime}>N-\beta$, that is $L_{7}<\beta$. Then we have

$$
L_{1-3}+L_{7}<(N-\theta)+\beta<N+\beta=L_{4-6}^{\prime} .
$$

Thus, lightpath 7 can be fit into the slot-cycle in the clockwise direction.

Property 10: Assume the length of all slot cycles is $2 N$ in a bidirectional ring. A single-loop logical topology (consisting of all nodes) can be constructed if $m \geq 2\lceil N / 7\rceil$ where $m$ is the frame size of the bidirectional OTDM ring.

Proof: Divide the total lightpaths into sets of adjacent lightpaths. By Property 9, we can put each set of seven lightpaths into a slot cycle of $2 N$ hops (in both directions). This means that we will use two slots to implement each set of seven lightpaths. Thus, in total, we need at most $2\lceil N / 7\rceil$ time slots to implement the virtual topology. 
Property 11: In the single slot-cycle ring architecture presented in Property 5, a single-loop logical topology (consisting of all nodes) can be constructed if $m \geq\lceil N / 4\rceil$, where $m$ is the frame size of the bidirectional OTDM ring.

Proof: The proof of Lemma 1 in [11] can be adapted for our problem here. Consider a single-loop logical topology consisting of the following adjacent connections: $\left\{\left(n_{0}, n_{1}\right),\left(n_{1}, n_{2}\right)\right.$ $\left., \ldots,\left(n_{N-1}, n_{N}\right),\left(n_{N}, n_{1}\right)\right\}$. Denote the number of hops required to route a particular call $\left(n_{i-1}, n_{i}\right)$ in the clockwise direction by $L_{i}$. The average number of hops, denoted by $\bar{L}$, required in the clockwise direction can be denoted by

$$
\bar{L}=\frac{\sum_{i=1}^{N} L_{i}}{N}
$$

In a single-loop topology, the requested lightpaths form an integer number of cycles around the ring. Therefore, the total length $\sum_{i=1}^{N} L_{i}$ must be a multiple of $N$, and $\bar{L}$ must be an integer.

Let $k=\left\lfloor N^{2} / 4 \bar{L}\right\rfloor$. It is shown in [11] that it is always possible to find a set of $k$ adjacent lightpaths with an average clockwise hop length $\tilde{L}$ less than or equal to $\bar{L}$. We call this set the clockwise set, and call the set of remaining connections the counterclockwise set. In the proposed architecture, there is only one slot cycle, and we place the clockwise set in the clockwise direction. The total hops required to accommodate the clockwise set is $k \tilde{L} \leq N^{2} / 4$. Since the slot cycle is $m N$, we can place the clockwise set in the ring if $m N \geq N^{2} / 4$ (or $m \geq\lceil N / 4\rceil$ ).

Next, we consider the remaining $(N-k)$ connections. Denote the average clockwise hop length $\check{L}$ of the set. Obviously, we have $\bar{L} \leq \check{L} \leq N$. If we route them through the counterclockwise ring, the average hop length must be $(N-\check{L})$. Let $D_{W}$ be the total number of contiguous hops required to accommodate the counterclockwise set. Then, we have

$$
\begin{aligned}
D_{W} & =(N-k)(N-\check{L}) \\
& \leq(N-k)(N-\bar{L}) \\
& =\left(N-\left\lfloor N^{2} / 4 \bar{L}\right\rfloor\right)(N-\bar{L}) .
\end{aligned}
$$

It can be shown that for integer $N$ and $\bar{L}$, the last quantity is maximized at $\bar{L}=N / 2$ [11]. This gives us

$$
D_{W}=\left(N-\left\lfloor N^{2} / 4 \bar{L}\right\rfloor\right)(N-\bar{L}) \leq N^{2} / 4 \text {. }
$$

Thus, $m(\lceil N / 4\rceil)$ time slots suffice to set up the counterclockwise set.

Properties 6, 10, and 11 show the space-reuse efficiencies of OTDM rings of different slot-cycle lengths. Since the bandwidth of each slot is the same in all cases, a smaller $m$ (frame size) means a smaller total bandwidth required to construct a singleloop virtual topology. As can be seen, a longer slot cycle usually leads to a higher efficiency of ring utilization. We can also see that the routing and slot assignment in a single-slot-cycle ring is much simpler than in rings with multiple slot cycles (see Properties 9 and 10).

\section{Space-Reuse EfFiciency: Traffic Model II}

In this section, we consider a more general traffic matrix. Again $T=\left[t_{i, j}\right]$ represents the traffic matrix and $t_{i, j}$ is the number of lightpaths to be set up from node $i$ to node $j$ in an OTDM ring. For simplicity, as in [13]-[16], we consider a unidirectional ring in this study where nodes are numbered from 0 to $(N-1)$ in the clockwise direction, where $N$ is the number of nodes in the ring. The load of $T$, denoted by $L(T)$, is the largest number of requested lightpaths carried by any link in the unidirectional ring [13]-[16]. Since the path is already determined in a unidirectional ring, the issue to tackle is to identify a time-slot assignment scheme to insert the lightpaths of $T$ into the slot cycles of the OTDM ring such that the bandwidth requirement is minimized for a given $L(T)$. We are going to use a generalized Tucker's algorithm [13], [14] as our time-slot assignment scheme.

\section{A. Generalized Tucker's Algorithm}

Assume $L(T)=L$. Without loss of generality, we assume that the load on every link is the same and equals $L$. If a link between node $u$ and node $(u+1)$ has a load $W<L$, we just add $(L-W)$ dummy lightpaths from $u$ to $(u+1)$ to make the total number of lightpaths passing through the link $=L$. Once the load is uniform on all links, the number of lightpaths terminating at a node equals the number of lightpaths originating from the node. If a node has no terminating lightpaths, neither will it have originating paths. A node of this type is called a nonterminating node in the paper.

We generalize Tucker's algorithm as follows. It consists of two steps: 1) index all the requested lightpaths in $T$ and 2) assign free slots to each indexed lightpath. Note that we add some dummy one-hop lightpaths in $T$ to create uniform loading. We can simply remove these dummy lightpaths after all lightpaths have been set up by the algorithm.

The Indexing Procedure: Let $A_{1}=\left(a_{1}, b_{1}\right)$ denote the first indexed lightpath in a uniform loading $T$. Select any lightpath as $A_{1}$. For $i=2, \ldots, w$, where $w\left(=\sum_{i} \sum_{j} t_{i, j}\right)$ is the total number of lightpaths in $T$, we move along the ring in the clockwise direction and identify the connection $A_{i+1}=\left(a_{i+1}, b_{i+1}\right)$ in $I \backslash\left\{A_{1}, A_{2}, \ldots, A_{i}\right\}$ by the following rule: $A_{i+1}$ is the first unindexed connection to begin after $b_{i}$ (the destination node of $A_{i}$ ). Since multiple lightpaths can start from the same node, we will arbitrarily pick one when that happens. We use $I$ to denote the set of indexed lightpaths, i.e., $I=\left\{A_{1}, A_{2}, \ldots, A_{w}\right\}$.

An immediate result of uniform loading is that lightpaths in $I$ form cycles: $C_{1}, C_{2}, C_{3}, \ldots, C_{v}$. A similar phenomenon is also found in a WDM ring [12]. This can be seen from the fact that the number of terminating lightpaths is the same as that of originating paths under uniform loading. $A_{1}$ terminates at $b_{1}$. So another path must originate from $b_{1}$. The indexing rule states that $A_{2}$ is the first lightpath starting after $b_{1}$, thus $a_{2}=b_{1}$ must hold. The same relationship exists between $A_{2}$ and $A_{3}$. The process continues until some $A_{j}$ 's destination node $b_{j}$ is the same as $a_{1}$ and a cycle is formed (such a lightpath must exist as $A_{1}$ originates from $a_{1}$ ). The next lightpath $A_{j+1}$ will start a new cycle and eventually every lightpath in $I$ is associated with a lightpath cycle.

For cycle $C_{k}$, we use the source node of its first lightpath as the reference point, denoted by $r_{k}$. For example, $r_{1}=a_{1}$ 
and $r_{2}=a_{l}$ if $C_{2}$ starts at $A_{l}$. Excluding the lightpaths in the first $j$ cycles, all nodes between $r_{j}$ and $r_{j+1}$ must be nonterminating nodes. Otherwise, $A_{j+1}$ will not be the first lightpath starting after $b_{j}\left(b_{j}=r_{j}\right)$. We use $g_{j}$ to denote the gap from node $r_{j}$ to $r_{j+1}$. Let $\left|g_{j}\right|$ be the gap size-the number of hops from node $r_{j}$ to $r_{j+1}$. Note that $\left|g_{j}\right|$ may be zero. Obviously, $\left|g_{j}\right|=\left(r_{j+1}-r_{j}\right) \bmod N$ and $\sum_{j=1}^{l}\left|g_{j}\right|=$ $\left(\left(r_{l+1}-r_{1}\right) \bmod N\right)<N$, for $1 \leq l<v$, where $v$ is the total number of cycles in $I$.

The Slot Assignment Scheme: Starting from $a_{1}$, we trace each slot cycle in the OTDM ring. Without loss of generality, we assume $a_{1}=0$. Otherwise, we can shift the node index and make $a_{1}=0$. We list the number of slots traced in each slot cycle. Each slot is denoted by a (node, slot number) pair in the paper. If we ignore the slot number and only list the node number, the result of the tracing is as follows:

$$
\begin{aligned}
& \mid \leftarrow \quad \text { cycle length }=k N \quad \rightarrow \mid \\
& 01 \ldots(N-1) 01 \ldots(N-1) 01 \ldots(N-1)(\text { slot-cycle } 1) \\
& 01 \ldots(N-1) 01 \ldots(N-1) 01 \ldots(N-1)(\text { slot-cycle } 2) \\
& \ldots \\
& 01 \ldots(N-1) 01 \ldots(N-1) 01 \ldots(N-1)(\text { slot-cycle } n)
\end{aligned}
$$

Starting from slot-cycle 1 , find a free shortest track from node $a_{1}$ to $b_{1}$ to set up $A_{1}$ (note that $a_{1}=0$ ). The word "shortest" will ensure that the length of the free track used is $<N$. We do the same and find a shortest track from $a_{i}$ to $b_{i}$ for $A_{i}$ in $I$ until the current slot cycle can no longer accommodate a new lightpath. We then move on to the next slot cycle.

Associated with the slot assignment algorithm is the concept of rounds. The slot assignment algorithm inserts the lightpaths one by one into slot cycles. We use $r_{1}$ as reference point and say that the algorithm has completed $p$ rounds if the algorithm traversed node $r_{1}(p+1)$ times, where lightpath $A_{1}$ is included in counting. Let $H_{p}$ be the set of lightpaths assigned during the $p$ th round. So, the lightpath set $I$ can also be divided into rounds $H_{1}, H_{2}, \ldots, H_{\lambda}$, where $\lambda$ is the total number of rounds in $I$. Let $B_{p}$ be the last lightpath in $H_{p} . B_{p}$, by definition, traverses node $r_{1}$. But, as stated in the following property, it must traverse $r_{u}$ if $B_{p}$ belongs to $C_{u}$.

Property 12: Suppose $B_{p}$ belongs to $C_{u}$. Then $B_{p}$ traverses (ends at or passes) node $r_{u}$.

Proof: This is due to the fact that all nodes from $r_{1}$ to $r_{u}$ are nonterminating nodes for $C_{u}$.

\section{B. Performance Analysis of the Algorithm}

To show the efficiencies of OTDM rings with different slotcycle lengths, we need to introduce some properties first. Let $I_{p}=\cup_{i=1}^{p} H_{i}$ be the set of all lightpaths assigned by the algorithm up to the $p$ th round.

Property 13: Let $R_{p}$ be the total length of a free track needed to accommodate all the lightpaths in $I_{p}$. Then, $p N \leq R_{p} \leq$ $(p+1) N$.

Proof: Assume the free track starts from node 0 because $A_{1}$ starts from node 0 . We insert lightpaths into the free track one by one. As there is no gap between two lightpaths unless they belong to different cycles; hence, we have $R_{p}=\left|I_{p}\right|+$ $\sum_{i=1}^{u-1}\left|g_{i}\right|$, where $B_{p}$ belongs to $C_{u}$, and $\left|I_{p}\right|$ is the total number of links traversed by lightpaths in $I_{p}$. Since $B_{p}$ traverses $r_{u}$ (Property 12), we have

$$
\left|I_{p}\right| \leq p N+\left(N-r_{u}\right)
$$

where $\left(N-r_{u}\right)$ is the maximum number links after $r_{u}$ that can be traversed by $B_{p}$. This leads to

$$
\begin{aligned}
\left|I_{p}\right| & +\sum_{i=1}^{u-1}\left|g_{i}\right| \\
& \leq p N+\left(N-r_{u}\right)+\left(\left(r_{u}-r_{u-1}\right)+\cdots+\left(r_{2}-r_{1}\right)\right) \\
& =(p+1) N-r_{1} \\
& =(p+1) N .
\end{aligned}
$$

Note that $r_{1}=0$ and $\left|I_{p}\right|+\sum_{i=1}^{u-1}\left|g_{i}\right| \geq p N$ is trivially true. We, thus, prove $p N \leq R_{p} \leq(p+1) N$.

Property 14: We can set up all the lightpaths of $I_{k-1}$ into a slot-cycle length of $k N$, where $k \geq 2$.

Proof: By Property 13, the total segment length needed to assign all the lightpaths in $I_{k-1}$ is less than $k N$. So a slot cycle of length $k N$ can accommodate all the lightpaths of $I_{k-1}$.

Let $L(I)$ represent the load created by lightpaths in $I$, that is, $L(I)$ is the maximum number of lightpaths passing through a link.

Property 15: For any given $p, L\left(I \backslash I_{p}\right) \leq L(I)-p$, where $L\left(I \backslash I_{p}\right)$ is the load created by the set of lightpaths in $\left(I \backslash I_{p}\right)$.

Proof: Suppose $B_{p}$ belongs to lightpath-cycle $C_{u}$. When $B_{p}$ terminates at node $r_{u}$, the lightpaths of the first $p$ rounds form exact $u$ cycles: $I_{p}=\cup_{i=1}^{p} H_{i}=\cup_{i=1}^{u} C_{u}$. Thus $L\left(I_{p}\right)=p$. When $B_{p}$ passes node $r_{u}, L\left(I_{p}\right)$ is larger than $p$. Thus, the condition $L\left(I \backslash I_{p}\right) \leq L(I)-p$ must hold.

Property 16: Let $T$ be a traffic matrix with the load constraint $L(T)=L$. If the slot-cycle length is $k N$ and $k \geq 2$, then $(k\lceil L /(k-1)\rceil)$ time slots (i.e., the frame size) suffice to set up all the lightpaths in $T$.

Proof: We first use Tucker's algorithm to index all lightpaths and create the set $I$. Property 14 shows that we can put the lightpaths of the first $(k-1)$ rounds into one slot cycle. Property 15 indicates that the remaining lightpaths will have a load $\leq(L(I)-(k-1))$. Thus, by repeating the generalized Tucker's algorithm $\lceil L /(k-1)\rceil$ times, we can set up all the remaining lightpaths in $I$. Each slot cycle use $k$ slots in the OTDM frame. Thus $(k\lceil L /(k-1)\rceil)$ time slots (i.e., the frame size) suffice to set up all lightpaths of a traffic matrix with load $=L$.

Property 16 indicates that the space-reuse efficiency of the proposed OTDM rings under the class of traffic matrices discussed in this section is also affected by the slot-cycle length of the ring. The longer the slot-cycle length, the better the spacereuse efficiency we can achieve. This is consistent with the results derived in Section III. 


\section{CONCLUSION}

We have presented a novel TSI-free OTDM ring architecture. We showed that all the slots in a TSI-free OTDM ring will form a cycle and all slot cycles must have the same length. The performance of the ring is related to its slot-cycle length. We showed that by exploring the algebraic relationship between the frame size and the total propagation delay of the ring, we can design a TSI-free OTDM ring with the longest slot-cycle length. To achieve the same result in a WDM ring, however, wavelength converters must be used. This innovative ring architecture can simplify the optical buffer requirement and reduce the implementation cost. It is a more practical architecture choice for a real implementation of OTDM rings.

\section{REFERENCES}

[1] N.-F. Huang, G.-H. Liaw, and C.-P. Wang, "A novel all optical transport network with time-shared wavelength channels," IEEE J. Sel. Areas Commun., vol. 1, no. 10, pp. 1863-1875, Oct. 2000.

[2] B. Wen and K. M. Sivalingam, "Routing, wavelength and time-slot assignment in time division multiplexed wavelength-routed optical wavelength networks," in Proc. IEEE INFOCOM 2002, New York, pp. 1442-1450.

[3] A. Chen, A. Wong, and C. T. Lea, "Routing and time-slot assignment in optical TDM networks," IEEE J. Sel. Areas Commun., vol. 22, no. 9, pp. 1648-1657, Nov. 2004.

[4] S. Subramanian, E. Harder, and H.-A. Choi, "Scheduling multi-rate sessions in time division multiplexed wavelength-routing networks," IEEE J. Sel. Areas Commun., vol. 18, no. 10, pp. 2105-2110, Oct. 2000.

[5] B. Li, Y. Qin, X.-R. Cao, and K. M. Sivalingam, "Photonic packet switchings: Architecture and performance," SPIE Opt. Netw., vol. 2, pp. 27-39, Jan./Feb. 2001

[6] J. Y. Wei, "Advances in the management and control of optical Internet," IEEE J. Sel. Areas Commun., vol. 20, no. 4, pp. 768-785, May 2002.

[7] S. Yao, B. Mukherjee, and S. Dixit, "Advances in photonic packet switching: An overview," IEEE Commun. Mag., vol. 38, no. 2, pp. 84-94, Feb. 2000.

[8] B. Meagher, G. K. Chang, G. Ellinas, Y. M. Lin, W. Xin, T. F. Chen, X. Yang, A. Chowdhury, J. Young, S. J. Yoo, C. Lee, M. Z. Iqbal, T. Robe, H. Dai, Y. J. Chen, and W. I. Way, "Design and implementation of ultralow latency optical label switching for packet-switched WDM networks," J. Lightw. Technol., vol. 18, pp. 1978-1987, Dec. 2000.

[9] P. C. Ku, C. J. Chang-Hasnain, J. Kim, and S. L. Chuang, "Variable optical buffer using slow light in semiconductor nanostructures,", in Proc. IEEE, Special Issue Nanoelectronics Nanoscale Process., Nov. 2003, vol. 91, pp. 1884-1897.

[10] A. Narula-Tam, P. J. Lin, and E. Modiano, "Efficient routing and wavelength assignment for reconfigurable WDM networks," IEEE J. Sel. Areas Commun., vol. 20, no. 1, pp. 75-88, Jan. 2002.

[11] L.-W. Chen and E. Modiano, "Efficient routing and wavelength assignment for reconfigurable WDM networks with wavelength converters," IEEE/ACM Trans. Netw., vol. 13, no. 1, pp. 173-186, Feb. 2005.

[12] R. Ramaswami and G. Sakaki, "Multi-wavelength optical network with limited wavelength conversion," IEEE/ACM Trans. Netw., vol. 6, no. 6, pp. 744-754, Dec. 1998.

[13] A. Tucker, "Coloring a family of circular arcs," SIAM J. Appl. Math., vol. 29, pp. 493-502, Nov. 1975.

[14] M. Valencia-Pabon, "Revisiting Tucker's algorithm to color circular arc graphs," SIAM J. Comput., vol. 32, pp. 1067-1072, 2003.

[15] O. Gerstel, G. Sasaki, S. Kutten, and R. Ramaswami, "Worst-case analysis of dynamic wavelength allocation in optical networks," IEEE/ACM Trans. Netw., vol. 7, no. 6, pp. 833-845, Dec. 1999.

[16] G. Li and R. Simha, "On the wavelength assignment problem in multifiber WDM star and ring networks," IEEE/ACM Trans. Netw., vol. 9, no. 1, pp. 60-68, Feb. 2001.

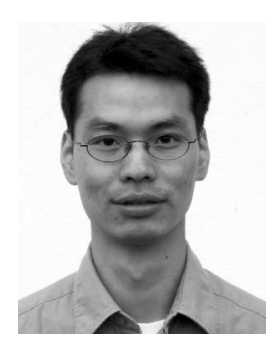

An Chen received the B.S. degree in electronic engineering from Fudan University, Shanghai, China, in 2001 and the Ph.D. degree in electronic engineering from the Hong Kong University of Science and Technology, Kowloon, Hong Kong, in 2007.

Currently, he is a software engineer at Giant Innovation, Hong Kong. His current research interests include optical network design and analysis and buffer management in wavelength-division multiplexing (WDM)/time-division multiplexing (TDM) systems.

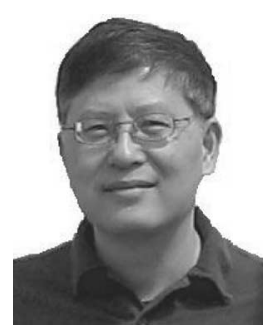

Chin-Tau Lea received the B.S and M.S. degrees from the National Taiwan University, Taipei, Taiwan, in 1976 and 1978, respectively, and the Ph.D. degree from the University of Washington, Seattle, in 1982, all in electrical engineering.

He is currently a Professor at the Hong Kong University of Science and Technology (HKUST), Kowloon, Hong Kong, which he joined in 1996. Prior to that, he was with AT\&T Bell Laboratories from 1982 to 1985 and with the Georgia Institute of Technology from 1985 to 1995 . His current research interests include switching and networking. He is the holder of five U.S. patents.

Dr. Lea is on the editorial board of IEEE JSAC and of Computer Networks. He received the DuPont Young Faculty Award from Georgia Tech in 1987 the IEEE Jack Neubauer Paper Award in 1998, and the School of Engineering Teaching Award from HKUST in 1998.

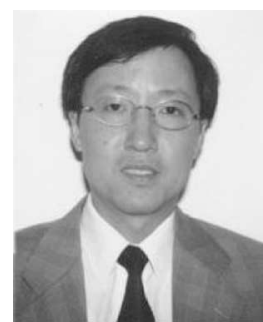

Albert Kai-Sun Wong received the S.B., S.M., E.E., and Ph.D. degrees in electrical engineering, all from the Massachusetts Institute of Technology, Cambridge, in 1982, 1984, and 1988, respectively.

He is currently a Visiting Associate Professor and Executive Director of Nansha Development at the Hong Kong University of Science and Technology, Hong Kong. From 1988 to 2000, he was with AT\&T and Lucent Technologies/Bell Laboratories, holding a series of positions as Member of Technical Staff, Distinguished Member of Technical Staff, Technical Manager, Director of Technical Marketing, and Director, Sales and Technical Marketing. His work at Bell Laboratories spanned from basic research, product development, to marketing and sales. From 2000 to 2001, he was Chief Operating Officer of Transtech Services Group. From 2002 to 2004 he was a Vice President of the Applied Science and Technology Research Institute of Hong Kong. He also held visiting and adjunct faculty positions at the Chinese University of Hong Kong, Polytechnic University of New York, and Rutgers University. His current research interests include communication networks, performance analysis, and photonic and data switching systems. 